Recepción: 03/06/2015

Evaluación: 16/06/2015

Aprobación: 20/08/2015

Artículo de Investigación Científica

\title{
LA EVALUACIÓN COMO EJERCICIO DEL PODER DOCENTE
}

\author{
Omaira Elisabeth Pejendino Piandoy \\ Universidad de Nariño \\ omairapejendino@gmail.com \\ Roberto Hernando Piandoy Jojoa \\ Universidad de Nariño \\ robertopiandoy@gmail.com
}

\begin{abstract}
RESUMEN
El presente artículo, es resultado de la investigación titulada: "El ejercicio del poder docente en la evaluación de los aprendizajes"1; se propone comprender el sentido pedagógico del ejercicio del poder docente en la evaluación de los aprendizajes; teniendo como escenario principal, el contexto de enseñanza-aprendizaje de la Institución Educativa Simón Bolívar del Municipio de Samaniego. Para el logro de este objetivo se realiza una aproximación al concepto de evaluación, de igual manera, se rastrean las diferentes concepciones y manifestaciones del ejercicio del poder que se producen en el aula de clase.

La metodología utilizada para el desarrollo de este propósito es la indagación bibliográfica, la entrevista a profundidad, la sistematización conceptual, el análisis de la información y las perspectivas teóricas de los diferentes autores consultados.

Como resultado de esta investigación se puede señalar que: la evaluación se entiende como un proceso integral, en la cual subyace el ejercicio del poder docente, que debe ser comprendido y ejercido con un sentido pedagógico con el fin de lograr resultados positivos en el contexto de los procesos educativos.
\end{abstract}

Palabras clave: Evaluación, ejercicio del poder docente, enseñanza, aprendizaje.

1. La investigación se realizó como trabajo de grado en la Maestría en Educación. 


\title{
EVALUATION AS AN EXERCISE FOR TEACHER EMPOWERMENT
}

\begin{abstract}
This article is part of the degree paper: "The exercise of the teacher's power in the evaluation of learning," which intends to understand the pedagogical meaning of the exercise of the teacher's power in the evaluation of learning; having as its main principle the context of teaching-learning in the Simon Bolivar Educational Institute in the municipality of Samaniego. The achievement of this objective necessarily incorporates an approach to the concept of evaluation; in the same way, it shows how different conceptions and expressions of the exercise of power are produced in the classroom.

The methodology used for the development of this purpose incorporates bibliographic inquiry, in-depth interviews, conceptual systematization, analysis of the information, and the theoretical perspectives of different authors who have been consulted.

The result of this researching is to point out that evaluation is understood as a comprehensive process underlying the empowerment of the teacher, which should be understood and exercised in a pedagogical sense in order to achieve positive results in the context of the educational process.
\end{abstract}

Key words: evaluation, exercise to empower the teacher, teaching and learning.

\section{A AVALIAÇÃO COMO EXERCICIO DO PODER DO DOCENTE}

\begin{abstract}
RESUMO
Este artigo, faz parte da pesquisa: "O exercício do poder docente na avaliação da aprendizagem"; Destina-se a compreender o sentido pedagógico do exercício do poder docente na avaliação do aprendizagem, com o cenário principal, o contexto de ensino-aprendizagem de município Escola Simon Bolivar de Samaniego. Para atingir este objectivo se realiza uma abordagem ao conceito de avaliação, da mesma forma, se rastreiam as diferentes concepções e manifestações do exercício do poder que ocorrem na sala de aula . A metodologia utilizada para o desenvolvimento deste objectivo é a investigação bibliográfica, entrevista em profundidade, a sistematização conceitual, o análise de dados e as perspectivas teóricas de diferentes autores consultados.

Como resultado desta pesquisa, pode-se notar que: a avaliação é concebida como um processo integral , que está na base do exercício do poder de ensino, que deve ser compreendido e exercido com um sentido pedagógico, a fim de alcançar resultados positivos no contexto da processos educativos.

Palavras-chave: Avaliação, exercicio do poder docente, ensino, aprendizagem.
\end{abstract}




\section{INTRODUCCIÓN}

El presente artículo de investigación, busca generar elementos conceptuales que permitan comprender el proceso evaluativo, revelando las manifestaciones del poder docente, las cuales son fruto de la dinámica de las relaciones de fuerza entre docentes y estudiantes. El empleo del concepto de evaluación y del poder docente en el discurso pedagógico permite abrir nuevos panoramas de significados lingüísticos que directa o indirectamente influyen en la realidad educativa, específicamente en la práctica docente.

Así, se realiza un análisis y reflexión en torno a la evaluación en relación con el ejercicio del poder docente, pues la evaluación no debe ser considerada un instrumento exclusivo para disciplinar o imponer el autoritarismo, en el cual aparecen de manifiesto las relaciones de dominación de unos sobre otros. El ejercicio del poder docente debe enriquecer el quehacer pedagógico, para hacer de la evaluación un proceso, que más que medir los conocimientos alcanzados, busque favorecer el aprendizaje.

De este modo, el artículo comprende una revisión de dos categorías principales: la evaluación y el ejercicio del poder docente, a partir de una aproximación conceptual a estos dos términos, toda vez que su análisis aporta a la comprensión del sentido pedagógico del ejercicio del poder docente en la evaluación de los aprendizajes, objetivo principal de la investigación a la que pertenece esta aproximación conceptual.

\section{Metodología}

Esta investigación se realiza desde el paradigma cualitativo, empleando el método etnográfico que está orientado por el concepto de cultura y tiende de manera generalizada a desarrollar conceptos y a comprender las acciones humanas desde un punto de vista interno ${ }^{2}$. Por consiguiente, en este trabajo se abordará la comprensión de los diversos significados de evaluación y poder, que se pueden desarrollar dentro de las aulas de clase; esto se realizará a través de un análisis construido desde la perspectiva de los informantes, sin negar la multiplicidad de las perspectivas teóricas, de los discursos, de los textos, de los contextos y de los afectos que se manifiestan en un espacio y en un tiempo determinado.

La presente investigación se desarrolla en la Institución Educativa Simón Bolívar del Municipio de Samaniego (Nariño), representada en un total de siete docentes. La selección de los docentes se realiza a través de un muestreo

2. Carlos Sandoval, La investigación cualitativa (Bogotá: Arfoeditores, 2002), 20. 
intencional, teniendo como criterio: que el docente debe llevar como mínimo 3 años de trabajo en la Institución Educativa; tener un nombramiento por término indefinido y ante todo contar con voluntad de colaboración e interés por el desarrollo del tema de investigación.

Se utilizan como instrumentos de recolección de información la entrevista a profundidad y el grupo de discusión. En la aplicación de la entrevista a profundidad se trabaja con cada uno de los entrevistados un cuestionario guía previamente elaborado. Posterior a la entrevista se hace la devolución de la información recolectada por medio de un documento impreso, sobre el cual el entrevistado podrá realizar modificaciones o sugerencias. En el caso del grupo de discusión, se reunió a los docentes con quienes se realizó la entrevista a profundidad y a otros docentes que quisieron participar, con el fin de profundizar en los temas más destacados.

\section{Planteamiento del problema}

Actualmente, el ejercicio de la docencia se ha tornado en una actividad muy compleja, llena de problemáticas y exigencias de toda índole por parte de los entes oficiales, la familia y la sociedad; hecho que ha centrado toda la atención en el trabajo desarrollado por los docentes en el aula. Al respecto, Martínez y Herrera mencionan que el aula de clase debe ser: "un escenario atractivo y recreador en donde los educandos disfruten el placer por aprender y por formarse: ese espacio físico a donde se asiste en búsqueda del conocimiento, el desarrollo de la habilidad y la destreza, al encuentro con los valores, en oposición a la anacrónica imagen de la misma, como un lugar no siempre deseable, signado por gobiernos escolares y métodos punitivos, discriminatorios, sin sentido ni significado con la vida"3.

Entre estos métodos punitivos, discriminatorios y a veces sin sentido ni significado para la vida del estudiante, se encuentra la evaluación de los aprendizajes, como una forma de calificación, clasificación y medio para ejercer poder. Mediante la evaluación, el docente ha logrado someter la conducta de los estudiantes infundiendo temor, haciendo real el fracaso y opacando el verdadero sentido de la evaluación, gracias al régimen de notas, regaños, sanciones y prohibiciones que se consideran legítimas porque tradicionalmente son parte de lo que se considera como ejercicio del poder docente.

Hay que tener en cuenta, que el papel del docente se ha visto envuelto en dificultades a causa del inadecuado ejercicio del poder, generando un impacto negativo, que en cierta medida ha deteriorado la imagen del docente y la de las instituciones educativas. Así, el actuar del docente se desarrolla entre dos

3. O. Martínez, y R. Herrera, Finalidades y alcances del Decreto 230 del 11 de febrero de 2002 (Bogotá: Enlace Editores Ltda.), 10. 
extremos, por un lado el facilismo y la permisividad que ha desembocado en la pérdida de valores y principios morales, además del incumplimiento de las normas por parte de los jóvenes, y por el otro lado, el autoritarismo al atentar contra el libre desarrollo de los estudiantes, al generar problemas emocionales tales como la timidez, introversión, sumisión y falta de autoestima.

En este sentido, algunos docentes han centrado su atención en la evaluación como una forma de medir los conocimientos de los estudiantes y controlar los actos de indisciplina en clase. Así cuando los estudiantes escuchan la palabra "evaluación", se sienten nerviosos, temerosos e intimidados, ya que la evaluación en este contexto implica la realización de un examen, sin sentido pedagógico que tiene como resultado bajas calificaciones.

De esta manera, el inadecuado ejercicio del poder por parte del docente, ha provocado que el ambiente del aula escolar no sea acorde con el espíritu de una educación para la formación integral de los estudiantes, ya que el docente se ha concentrado en "encontrar o diseñar la mejor prueba de evaluación posible, el tipo de evaluación ideal, como si ella efectivamente diera cuenta de la verdad sobre el rendimiento o no de los estudiantes"4.

En atención a lo anteriormente expuesto, se puede observar que el ejercicio del poder docente en la mayoría de las aulas de clase, no se está ejerciendo con un sentido pedagógico, y por esta razón no está contribuyendo a la realización de una evaluación para el aprendizaje, que permita alcanzar las metas propuestas en determinada área, nivel de estudios, periodo o grado de educación.

\section{Sobre el concepto de evaluación}

Evidentemente, la evaluación hace parte de la vida del ser humano, está presente en su diario vivir y constituye el camino para alcanzar y garantizar la calidad y excelencia de un proceso, una actividad o situación. Así, "La evaluación ha colonizado, el mundo de la vida, ha hecho presa de individuos, de instituciones, de poblaciones; ha pretendido medir, con patrones universales, sus acciones, sus proyectos, sus interacciones, su ser y su acontecer"5.

Ahora bien, el concepto de evaluación no ha sido único y perpetuo, teóricos, pedagogos, docentes, estudiantes, e incluso padres de familia, le han dado una definición según los propósitos y exigencias de la época y contexto institucional. Por lo cual, la evaluación ha tenido múltiples definiciones, como la de ser considerada una forma de calificar y clasificar o un proceso que

4. A. Ramíres, Evaluación de los aprendizajes y desarrollo institucional (Bogotá: ECOE, 2009), 22.

5. Tomas Sánchez Amaya, Aproximación a un estudio genealógico de la evaluación educativa en Colombia, segunda mitad del siglo XX. Tesis Doctoral. Doctorado en Ciencias Sociales. Niñez y Juventud Universidad de Manizales, 2008, 15. 
permite la valoración del aprendizaje. Así, la evaluación se puede entender de diversas maneras, dependiendo de las necesidades, propósitos u objetivos de la institución educativa, tales como: el control y la medición, el enjuiciamiento de la validez del objetivo, la rendición de cuentas, por citar algunos propósitos"6.

Dentro de este orden de ideas también se menciona que "Muchas veces el término "evaluación" (sinónimo de valoración) es utilizado como sinónimo de medida; dos términos distintos, aunque uno sea auxiliar del otro. Medir es comparar una propiedad física o no física de las personas o de las cosas, con la misma propiedad de otra cosa o patrón de comparación, que se toma como "unidad de medida", y determinar un número que indique cuantas veces más (o menos) que la unidad, posee el objeto medido dicha propiedad. Evaluar es interpretar, con base en algún criterio, los resultados de la medición y juzgar la calidad o el valor del aspecto evaluado"7.

De lo anterior, se puede entrever que la evaluación va más allá de la medición de los conocimientos y por lo tanto, implica un concepto más amplio y complejo, que incluye la parte subjetiva de los sujetos que participan en ella. Cuando un docente realiza el proceso evaluativo, "lo realiza para que el estudiante conozca con claridad acerca de los conocimientos que tiene, pero no solo para memorizarlos y que los repita, sino para ver que función tienen esos conocimientos dentro de su realidad; como los relaciona no solo con el saber hacer, sino en el saber convivir"8.

Así mismo, se menciona que la evaluación es "el proceso que facilita la toma de decisiones para proveer información al respecto de cómo están aprendiendo los estudiantes, cómo se está enseñando y cómo se puede mejorar. Al final de este proceso docentes y estudiantes juzgan si han logrado los objetivos de enseñanza y emiten un juicio de valor que permite tomar decisiones para intervenir los procesos, tanto de enseñanza como de evaluación de los aprendizajes" 9 .

Hay que destacar, que este concepto favorece una evaluación de corte participativo, puesto que los juicios de valor no se emiten arbitrariamente ni de manera vertical por parte del docente, ya que en conjunto se analiza los resultados obtenidos en el marco del proceso evaluativo, para estimar los

6. Ana Isabel Vargas, "La evaluación educativa: conceptos, periodos y modelos". Actualidades investigativas en Educación, vol. 4. No 2 (2004), 01-28.

7. Edmundo Calvache, La evaluación integral en los alumnos de los programas de licenciatura en la facultad de ciencias humanas de la Universidad de Nariño. Tesis especialización en/ Universidad Nariño (2005), 30.

8. Entrevista a Docente No. 8, Institución Educativa Simón Bolívar, Samaniego, Nariño, 21 de junio de 2014.

9. Omar Salazar Cabrales, "Contexto de la evaluación de los aprendizajes en la educación superior en Colombia: Sugerencias y alternativas para su democratización”. Revista Educación y Desarrollo Social. Vol. II - No. 01 (2008), 141-165. 
alcances y proponer estrategias para el mejoramiento. De igual manera, "la evaluación permite al docente identificar sus fortalezas y debilidades en el proceso evaluativo, las fortalezas para perfeccionarlas y las debilidades con el fin de corregirlas y convertirlas en aciertos y mejoras"10.

Por su parte, el Ministerio de Educación Nacional establece que: "la evaluación del aprendizaje de los estudiantes realizada en los establecimientos de educación básica y media, es el proceso permanente y objetivo para valorar el nivel de desempeño de los estudiantes"11. De esta manera, el Estado plantea una evaluación que involucra a todos los actores educativos como responsables de este proceso, estableciendo los deberes y derechos de los estudiantes para lograr una evaluación integral, que tenga en cuenta en todo momento los aspectos, académicos, personales y sociales.

Desde la perspectiva de Francisco Cajiao, la palabra evaluar tiene dos sentidos: "En primer lugar significa asignar valor a algo. Este significado primordial es fundamental desde el punto de vista pedagógico, ya que la evaluación escolar debería centrarse cada vez más en "descubrir" el valor de cada estudiante, la "identificación" de los talentos particulares, el "hallazgo" de las mejores formas de enseñar y de organizar las instituciones educativas. En segundo lugar significa identificar el progreso en el logro de unos objetivos propuestos, o averiguar el estado de algo con respecto a un parámetro (estándar) preestablecido. En general este es el sentido que se asigna con más frecuencia a la evaluación. Para muchos representa un sentido amenazante de calificación (o descalificación). Sin embargo, es necesario entender que todas las personas y las organizaciones humanas, por más informales que sean, siempre están recurriendo a este mecanismo, así lo hagan de una forma intuitiva"12.

Sin lugar a dudas en el ámbito escolar, se ha hecho énfasis en identificar el progreso académico de los estudiantes y se ha descuidado bastante el sentido de la valoración, posiblemente debido a que se requiere más esfuerzo por parte del docente para realizar una observación más detallada y sistemática, a la vez compleja porque posee una connotación más humana, que implica la participación, el trabajo en equipo y las relaciones interpersonales.

Por lo anterior, es importante asumir "el concepto de valoración para resaltar el carácter apreciativo de la evaluación y enfatizar que es ante todo

10. Entrevista a Docente No. 8, Institución Educativa Simón Bolívar, Samaniego, Nariño, 21 de junio de 2014.

11. República de Colombia, Decreto 1290 de 2009, artículo 01, Literal 3. (Bogotá: Ministerio de Educación Nacional, 2009).

12. Francisco Cajiao, Plan Decenal en acción: Evaluación del Aprendizaje y Calidad Educativa (Bogotá: Ministerio de Educación Nacional, 2008), http://cms-static.colombiaaprende.edu.co/cache/binaries/ articles-158486_archivo2.pdf?binary_rand=4009 (13 de agosto de 2013). 
un procedimiento para generar valor (reconocimiento) a lo que las personas aprenden, basado en la complejidad puesto que tienen en cuenta las múltiples dimensiones y relaciones entre los estudiantes, empresas y docentes"13.

No obstante, la gran cantidad de estudiantes por cada docente, hace que el tiempo y las fuerzas no sean suficientes para adelantar este proceso y sea difícil descubrir el verdadero valor de un estudiante, sus intereses, sus talentos, sus estilos de aprendizaje, dicho de otra manera, no se logra valorar la diferencia sino que se estandariza con una serie de pruebas.

Sin lugar a dudas, la única manera de evaluar masivamente es mediante la comparación estadística, cifras en las que no se tiene en cuenta la promoción humana sino el adiestramiento para el mercado de trabajo. Así, actualmente "la evaluación se realiza para ver si se está alcanzando los logros propuestos, que es lo principal" 14 .

De esta forma, el estudiante ha llegado a considerar la evaluación como "un mundo privado del maestro en tanto éste se identifica como sujeto que posee un conocimiento y una información, y controla al interior del aula los procesos que garantizan su reproducción. El maestro determina el tiempo, el espacio y las estrategias y se constituye así en el eje central de la misma. "El docente establece una fecha para hacer la evaluación y el estudiante tiene que prepararse y demostrar lo que se le enseño, y si se observa que les fue totalmente mal a los estudiantes, se refuerza para volver a hacer esa evaluación"15.

Al respecto de evaluación, Niño, Perafán y Carrillo mencionan que en la concepción cuantitativa, la "evaluación por objetivos", la "evaluación sin referencia a objetivos" y la "evaluación para la toma de decisiones", a pesar de sus evidentes diferencias metodológicas responden a una misma concepción autocrática que coloca a los agentes evaluadores en una relación de poder favorable, por la posibilidad de someter los sujetos evaluados a una relación desfavorable, caracterizada por el desconocimiento y sometimiento a los instrumentos y a la información manipulada por los primeros. No es difícil reconocer en la escuela, que este tipo de práctica legitima un orden jerárquico de poder donde la base de los sometidos la constituyen los estudiantes, sobre quienes recae todo el peso de la pirámide" 16 .

13. Sergio Tobón, Formación Basada en Competencias (Bogotá: Ecoe Ediciones Ltda., 2004), 09.

14. Entrevista a Docente No. 1, Institución Educativa Simón Bolívar, Samaniego, Nariño, 21 de junio de 2014.

15. Entrevista a Docente No. 2, Institución Educativa Simón Bolívar, Samaniego, Nariño, 21 de junio de 2014.

16. Liberia Carrillo, Libia Niño y Andrés Perafán, La evaluación: ¿instrumento de poder o acción cultural? (Bogotá: Universidad Pedagógica Nacional, 1996), http://www.pedagogica.edu.co/stogare/ps/ articulos/peda08_05eval.pdf (13 de agosto de 2013). 
En contraposición al concepto anterior, surge la evaluación para el aprendizaje definida como un tipo de evaluación cuyo propósito principal es mejorar el proceso de aprendizaje, a través de una revisión permanente de dicho proceso, utilizando diferentes instrumentos que permitan a los docentes determinar los pasos a seguir para fortalecer el aprendizaje de los estudiantes, y propiciar espacios de autorreflexión del aprendizaje en los estudiantes, para permitirles pensar las estrategias de aprendizaje que más lo benefician ${ }^{17}$.

Es preciso tener en cuenta, que dentro del proceso evaluativo el ejercicio del poder docente, en primer lugar, conlleva a entender la evaluación, como intimidación para mantener el manejo de grupo, y en segundo lugar, como proceso que se desarrolla para buscar estrategias de apoyo que permitan aprendizajes significativos.

Por último es conveniente, recalcar que a pesar de los múltiples conceptos de evaluación existentes, ésta se sigue aplicando en función de la verificación de los conocimientos adquiridos por el estudiante, prueba de ello, es que los docentes asimilan la evaluación con el examen, la prueba más difundida y aplicada en todo el sistema educativo.

Ejemplos en relación a lo anterior:

- "Pues ahorita se le llama evaluación pero es examen porque el docente quiere ver si el estudiante está aprendiendo o no, si se está alcanzando los logros o no. Entonces en esa parte diríamos que es un examen"18.

- "Los exámenes se los aplica por obligación porque la misma ley desde el Ministerio nos obliga a nosotros a hacer evaluaciones, evaluaciones saber para los grados 3, 5, 9 y 11, entonces estamos obligados a hacerlas"19.

\section{Sobre el concepto de poder}

El poder es un fenómeno social, histórico, político y psicológico, que desde mucho tiempo atrás ha despertado el interés de muchos pensadores, en diversos contextos, por esto, desentrañar el significado del poder, implica adentrarse en un mar de perspectivas.

Así, el poder es entendido como:

17. María Arranz y Victor Santiuste, Nuevas perspectivas en el concepto de evaluación (Madrid: Universidad complutense de Madrid, s.f.), http://www.revistaeducacion.mec.es/re350/re350_20.pdf (20 de junio 2014).

18. Entrevista a Docente No. 1, Institución Educativa Simón Bolívar, Samaniego, Nariño, 21 de junio de 2014.

19. Entrevista a Docente No. 3, Institución Educativa Simón Bolívar, Samaniego, Nariño, 21 de junio de 2014. 
Un "acto o instrumento en que consta la facultad que una persona confiere a otra para que, en lugar suyo y representándola, pueda ejercer un derecho o ejecutar alguna cosa. Tener expedita la facultad o potencia de hacer una cosa. Capacidad de controlar a otros en un ente y tener facultades decisorias a nivel de dirección. Posesión del control, autoridad e influencia sobre otros ${ }^{20}$.

Lo que sí es claro es que el poder subyace a la vida en sociedad, hasta tal punto que se puede considerar "que los hombres y las mujeres no son en esencia libres y que habitan un mundo repleto de contradicciones y asimetrías de poder y privilegios" 21 .

En Max weber el poder se entiende como "la posibilidad de imponer la propia voluntad sobre la conducta ajena dentro de una relación social, aun contra toda resistencia y cualquiera sea el fundamento de esa probabilidad"22, a su vez lo diferencia de los conceptos de dominación y disciplina, la primera entendida como la probabilidad de obediencia a un mandato y la segunda como la probabilidad de obediencia a un mandato de manera simple y automática, sin resistencia ni crítica.

Por su parte, los aportes de Foucault permiten entender al poder como "un conjunto de acciones sobre acciones posibles; opera sobre el campo de posibilidad o se inscribe en el comportamiento de los sujetos actuantes: incita, induce, seduce, facilita o dificulta; amplia o limita, vuelve más o menos probable; de manera extrema, constriñe o prohíbe de modo absoluto; con todo, siempre es una manera de actuar sobre un sujeto actuante o sobre sujetos actuantes, en tanto que actúan o son susceptibles de actuar. Un conjunto de acciones sobre otras acciones" 23 .

De esta forma, el poder que emana de las instituciones es un efecto de conjunto y además se ejerce por medio de ciertos dispositivos.

Así pues, las instituciones escolares, militares y hospitalarias tienen como base de funcionamiento los reglamentos, dispositivos con los cuales las personas pueden ser sometidas, utilizadas, transformadas o perfeccionadas, configurando lo que Foucault denomina los cuerpos dóciles.

Según Van Dijk, el poder es una característica de la relación social entre grupos en el cual los miembros de un grupo son capaces de controlar a los miembros de otro grupo, esto involucra el control de las acciones del otro ${ }^{24}$.

20. María Laura Valletta, Diccionario Jurídico (Buenos Aires: Valletta Ediciones, 2006), 641.

21. Peter Mclaren, La vida en las escuelas (Madrid España: Siglo XXI Editores, 1989), 203.

22. Max Weber. Economía y Sociedad (Madrid: Fondo de Cultura Económica. 1964), 1072.

23. Michel Foucault, "El sujeto y el poder". Revista Mexicana de Sociología, vol. 50. No. 3 (1988), 2-20.

24. Teun Van Dijk, Ideología una aproximación disciplinaria ( Barcelona: Gedisa, 2000), 120. 
Por lo tanto el ejercicio del poder se hace necesario, para que un grupo humano pueda sobrevivir en el medio natural, social y cultural, ya que, el poder actúa como elemento cohesionador de las acciones y potencialidades de los individuos para la consecución de unos propósitos que individualmente serían imposibles de alcanzar. Sucede pues que, el poder se delega a una persona, aun grupo de personas o una institución con el fin de orientar las acciones individuales.

En este sentido, tiene razón Hanna Arendt al afirmar que "el poder surge entre los hombres cuando actúan juntos y desaparece en el momento en que se dispersan" 25 .

Es preciso señalar que el poder tiene unas características, que dependiendo de la manera cómo se lo ejerza, generan lo que podríamos llamar unos tipos de poder.

En primer lugar el poder destructivo, que genera unos efectos siniestros, el cual es ejercido por los grupos militares y desde el cual se ha generado un concepto negativo del poder.

En segundo lugar, tenemos el poder productivo, el cual se manifiesta en los grupos empresariales.

Y en tercer lugar el poder integrador, que se da a nivel de los grupos sociales y del cual se desprende un sentido positivo del poder, ya que se manifiesta como una estrategia de apoyo para el progreso, que promueve la integración y permite que las organizaciones y los grupos sociales adquieran conductas de fraternidad, respeto y cooperación.

En relación con la educación de los niños y niñas, el poder juega un papel muy importante, ya que se requiere de la participación de todos los sujetos sociales, instituciones y grupos sociales, para ello se ha designado unas instituciones y unas personas para que se pongan al frente y son quienes realizan el ejercicio del poder, desplegando sus conocimientos adquiridos, experiencia y la autoridad para lograr movilizar las potencialidades de quienes se están educando.

Ahora bien, ¿Cómo es entendido el poder entre los docentes?, para abordar este interrogante es preciso mencionar que en la práctica docente, subyace el ejercicio del poder, y se hace más perceptible en el escenario del proceso evaluativo. Bourdieu y Passeron, afirman que: "en la escuela se reproduce la cultura dominante, contribuyendo así a reproducir la estructura de las relaciones de fuerza, en una formación social en que el sistema de enseñanza

25. Hanna Arendt, La Condición Humana (Barcelona: Paidos Ibérica S. A, 2005), 22. 
dominante tiende a reservarse el monopolio de la violencia simbólica legitima"26.

Desde la perspectiva de los docentes, se configuran unas concepciones de poder fruto de sus conocimientos y experiencias. "Yo entiendo el poder del docente como la autoridad, que uno tiene dentro de las funciones docentes, el poder está implícito en la labor del docente porque uno es una autoridad para los estudiantes, en las manos de uno tiene ese poder para manejar el grupo, es decir todos los aspectos académicos, disciplinarios, el reglamento mismo, el manual de convivencia, esas son las herramientas con que uno lo ejerce el poder con los estudiantes. Y por otro lado uno tiene el poder docente en las decisiones de la institución, uno tiene poder para decidir porque tiene voz y voto, es una parte integrante de la comunidad educativa y por tanto uno puede lograr un cambio porque tiene ese poder implícito en el docente"27

"Para mí el poder docente es la autoridad que uno tiene para desempeñar la labor, para poder llegar a los estudiantes"28.

Las anteriores apreciaciones, identifican el poder con la autoridad, la cual se considera connatural a la función docente, que le permite manejar la conducta y las acciones de todo un grupo, tomar decisiones y lograr cambios. Las principales decisiones del docente giran principalmente en torno a los aspectos académicos y disciplinarios, fundamentándose básicamente en el manual de convivencia institucional.

Generalmente, siempre se ha reconocido la autoridad que ejerce un docente sobre los estudiantes, ya que socialmente el docente goza de un prestigio, un conocimiento, unas técnicas y unos valores que le dan cierta capacidad de influir sobre los demás, sean estos directivos docentes, padres de familia y especialmente estudiantes; además, "la acción pedagógica implica necesariamente como condición social de su ejercer, la autoridad pedagógica y la autonomía relativa de la instancia que tiene la tarea de ejercerla"29.

Para aproximarnos al concepto de autoridad, es preciso retomar a Max Weber para quien la autoridad implica poder de mando y deber de obediencia, que son dos aspectos en los cuales por mucho tiempo se ha enmarcado la función del docente ${ }^{30}$.

26. Pierre Bordieu y Jean Passeron. La reproducción: elementos para una teoría del sistema de enseñanza (México: Fontamara, 1995), 46.

27. Entrevista a Docente No. 5, Institución Educativa Simón Bolívar, Samaniego, Nariño, 21 de junio de 2014.

28. Entrevista a Docente No. 02, Institución Educativa Simón Bolívar, Samaniego, Nariño, 21 de junio de 2014.

29. Bordieu y Passeron, La reproducción, 52.

30. Max Weber, Economía y sociedad, 623. 
Mandar y obedecer, dentro del ambiente escolar son aspectos que aunque no lo parezca preocupan mucho en el ámbito de la enseñanza aprendizaje, estas relaciones de fuerza, siempre persisten y generan contradicciones en la relación docente estudiante. La tendencia es obtener el mando, el respeto e influir en la conducta de los estudiantes, así lo dan a entender los docentes consultados:

"El poder docente es como el carácter del docente, la forma como él trata a los estudiantes, es la forma de hacerse respetar de hacerse entender para que el estudiante se comporte de X o Y forma" 31 .

“Huy es grandísimo el poder que tenemos! Porque con el poder docente nosotros somos capaces de influenciar la voluntad de estos chicos, eso es el poder, sí, en que somos capaces nosotros de hacer que ellos hagan lo que uno quiera y uno lo puede lograr de muchísimas maneras, sea con autoridad, sea con autoritarismos, sea como amigo, o sea el poder que nosotros ejercemos sobre estos jóvenes es inmenso y ahí de pronto es donde se desborda no, se desborda por los lado equivocados, entonces somos capaces de manejar la conducta de ellos, ese es el poder que podemos ejercer y ... se vuelven ellos como algo maleable, que lo puedo amoldar como yo quiera" ${ }^{32}$.

"El poder también es el dominio de grupo que todos estén atendiendo lo que uno está diciendo"33.

Para comprender lo anterior, es importante considerar los aportes de Arendt, quien argumenta que la autoridad excluye el uso de medios externos de coacción, por lo tanto se usa la fuerza cuando la autoridad fracasa, a su vez la autoridad implica una jerarquía admitida como legítima tanto por el que manda, como por el que obedece ${ }^{34}$.

Por lo tanto la dominación conlleva el uso de la fuerza y toda forma de violencia y esta no puede ser contemplada como una opción por el docente. Ya que existen otros medios para instaurar autoridad, por ejemplo el papel de enseñante le da una superioridad que se manifiesta de manera natural.

Bourdieu y Passeron hacen referencia respecto a la configuración de esta jerarquía mencionando que:

31. Entrevista a Docente No. 04, Institución Educativa Simón Bolívar, Samaniego, Nariño, 21 de junio de 2014.

32. Entrevista a Docente No. 06, Institución Educativa Simón Bolívar, Samaniego, Nariño, 21 de junio de 2014.

33. Entrevista a Docente No. 02, Institución Educativa Simón Bolívar, Samaniego, Nariño, 21 de junio de 2014.

34. Hanna Arendt, Qué es la autoridad parte I (s.c. 2011) http://es.scribd.com/doc/53732515/ArendtHannah-Que-Es-La-Autoridad-Parte-I (10 de septiembre de 2014). 
La autoridad pedagógica imprime un sello tan intenso en todos los aspectos de la relación de comunicación pedagógica que esta relación es frecuentemente vivida o concebida como el modelo de la relación primordial de comunicación pedagógica, o sea, la relación entre padres e hijos o, de modo más general, entre generaciones. La tendencia a restaurar en toda persona investida de una autoridad pedagógica la relación arquetípica con el padre es tan fuerte que todo aquel que enseña, por joven que sea, tiende a ser tratado como un padre ${ }^{35}$.

Así, "El profesor, como funcionario de un SE (sistema escolar), no necesita fundar su AuP (autoridad pedagógica) por su propia cuenta, en cada ocasión y en cada momento, porque, a diferencia del profeta o del creador intelectual, autores cuya autoridad depende de las intermitencias y las fluctuaciones de la relación entre el mensaje y las esperanzas del público, predica a un público de fieles confirmados, en virtud de la AuE (autoridad escolar), legitimidad de función que le garantiza la institución y que es socialmente objetivada y simbolizada en los procedimientos y reglas institucionales que definen la formación, los títulos que la sancionan y el ejercicio legítimo de la profesión"36.

También, se encuentra la autoridad que proviene del carisma del docente, otra afirmación de los docentes expresa lo siguiente.

"Es la capacidad del docente para manejar un grupo y es también la autoridad que se gana el docente con los hechos, con su profesionalismo en el cargo, con el respeto que se ha ganado de los estudiantes por su buen desempeño docente en todos los aspectos" 37 .

En el campo de la educación, el poder también opera encubriendo realidades, ocultando las relaciones de poder y sus objetivos, además impone, unos significados, una verdad que legitima la autoridad que es delegada a las instituciones, las cuales funcionan sin necesidad de hacer explicitas las formas más primitivas del poder, como son los castigos físicos, la reclusión o cualquier forma de violencia. Es decir que se ha creado una autoridad que se considera legítima sobre la base de una verdad que ha sido impuesta, arbitraria y que tiene la capacidad de someter las voluntades sin necesidad de recurrir a la fuerza.

Sobre este tipo de poder, Bourdieu y Passeron mencionan que "todo poder de violencia simbólica, es decir, todo poder que logra imponer significados e imponerlos como legítimos disimulando las relaciones de fuerza en las que se

35. Bordieu y Passeron. La reproducción, 59.

36. Bordieu y Passeron. La reproducción, 105.

37. Entrevista a Docente No. 01, Institución Educativa Simón Bolívar, Samaniego, Nariño, 21 de junio de 2014 
basa su fuerza, agrega su propia fuerza, es decir, una fuerza específicamente simbólica, a estas relaciones de fuerza"38.

Sucede pues que, en el ámbito de las relaciones sociales el poder es una fuerza, que mediante la imposición de unos significados, genera unas creencias que legitiman la obediencia a las instituciones o hacia unas personas permitiendo a su vez el control de la mente y del cuerpo de los sujetos que hacen parte de un determinado grupo social.

\section{Manifestaciones del poder docente en la evaluación de los aprendizajes}

En el contexto de las aulas de clase, el papel que desempeña el docente como profesional de la educación es fundamental, ya que alrededor de él gira todo el proceso educativo, pero principalmente el de enseñanza-aprendizaje. Por lo cual, la docencia más que una profesión, es una actividad social, una vocación representada en el servicio a la sociedad, que se desarrolla colectivamente a través de la relación de todos los integrantes de la comunidad educativa, directivos docentes, docentes, estudiantes, padres de familia y administrativos, que bajo la creación de espacios propicios para el conocimiento, buscan el fortalecimiento de los mismos y la aparición de nuevos saberes que surgen a través de la investigación. Pero también el desarrollo de valores, de actitudes y aptitudes que le permitan sobresalir en mundo actual.

Sin embargo, se debe tener en cuenta que "la tarea del maestro es distinta en los diferentes contextos sociales y geográficos de un país tan diverso y con tantas contradicciones como el nuestro. No es lo mismo trabajar en el centro que hacerlo en la periferia de las ciudades; no es lo mismo trabajar en contextos sociales relativamente estables que en lugares en donde se viven las tensiones propias de la violencia; no es lo mismo trabajar con alumnos que cuentan con todos los recursos que hacerlo en condiciones de enorme pobreza"39. Así, el docente de hoy en día debe asumir con gran responsabilidad el reto de formar seres humanos capaces de afrontar las nuevas exigencias de la sociedad, empezando por reconocer que la clase se convierte en ese espacio privilegiado para generar en los estudiantes espacios de reflexión, a partir del trabajo de temas que se relacionen con la cotidianidad del estudiante.

Por ende, el aula de clase es el espacio donde el ejercicio del poder docente empieza a tomar mayor importancia, al influir de manera directa en el proceso de enseñanza aprendizaje; un lugar donde "el poder se magnifica en la autoridad que da el saber enseñar, aprender y evaluar, en el conocimiento

38. Bordieu y Passeron, La reproducción, 200.

39. Carlos Hernández, eds. Para ejercer el oficio de maestro (Bogotá: Ministerio de educacion Nacional, 2005), http://www.mineducacion.gov.co/1621/article-87110.html (20 de mayo de 2015). 
profundo sobre lo que se enseña y en el reconocimiento del otro como un interlocutor válido, que es diferente y se respeta"40.

Generalmente, la primera manifestación del poder docente es la autoridad que se ejerce sobre los estudiantes y el proceso evaluativo, ya que socialmente el docente goza de un prestigio, un conocimiento, unas técnicas y unos valores que le dan cierta capacidad de influir sobre los demás; "la acción pedagógica implica necesariamente como condición social de su ejercer, la autoridad pedagógica y la autonomía relativa de la instancia que tiene la tarea de ejercerla"41.

Cajiao afirma que la institución escolar sigue siendo tan autoritaria como antes, ya que ha puesto en el maestro un poder absoluto sobre los niños, por lo tanto el respeto a la autoridad del docente es incuestionable ${ }^{42}$. Así, el ejercicio del poder proporciona al docente esa capacidad de desarrollar la autoridad sin necesidad de utilizar medios de fuerza que impliquen el sometimiento del otro: sabiendo que necesariamente la autoridad implica una jerarquía admitida tanto por él que manda, como por él que obedece.

Por otra parte, la autoridad tiene su fundamento en el aspecto psicológico del profesor, que está directamente relacionado con el dominio de las emociones y la forma como interactúa con sus estudiantes. "El profesor simpático tiene una autoridad que se impone naturalmente, en este caso no necesita recurrir a sanciones, amenazas o castigos. Ya que su autoridad es consecuencia, sobre todo, de su dominio sobre sí mismo en profundidad, en su inconsciente, donde controla la agresividad y la angustia. Son raros los casos de docentes simpáticos faltos de disciplina y autoridad"43.

Así, en la autoridad como manifestación del ejercicio del poder docente, la jerarquización siempre va a estar presente, ya que tradicionalmente el docente es superior al estudiante al tener un dominio del conocimiento y ser depositario de unos valores morales y sociales, que lo hacen merecedor de ser escuchado, respetado y obedecido.

Por lo tanto, "el estudiante sólo acepta la autoridad del docente cuando él sabe que el docente tiene el conocimiento y dice la verdad, y este reconocimiento se hace a través de la comunicación con el docente o la observación de su obrar,

40 Martha Lorena Salinas, eds. Conflictos y tensiones que merecen debatirse (Bogotá: Ministerio de Educación Nacional, 2008), http://www.mineducacion.gov.co/1621/article-162340.html (18 de mayo de 2014).

41. Bordieu y Passeron, La reproducción, 52.

42. Francisco Cajiao, La formación de maestros y su impacto social (Bogotá: Magisterio, 2004), 28.

43. Izquierdo, Moreno. El profesor y su mundo. Guías para maestros y profesionales de la educación (México: Editorial Trillas, 2002), 14. 
es decir su ejemplo"44. Así, cada una de las acciones que realiza el docente en su labor educativa, marca la autoridad que él tendrá frente a su grupo de estudiantes; "Las palabras convencen pero el ejemplo arrastra; cómo le pido a un niño que llegue puntual cuando siempre llego tarde a la clase, como le pido responsabilidad si falto constantemente a mi trabajo" 45 .

En consecuencia, el dominio del conocimiento por parte del docente, es otra manifestación del ejercicio del poder, de ahí, la importancia de la formación permanente, las investigaciones y actualizaciones en el área del conocimiento en que trabaja, ya que dará mayor seguridad al docente y motivara al estudiante. En particular la imagen profesional del docente se fortalece ya que deleitarse con un discurso sincero, rico en argumentos, ejemplificado y mezclado con historias de vida, aplicable a la realidad y puesto en escena con el humor e ingenio de un maestro, es lo que hace de la clase algo único y extraordinario que dignifica la labor del docente y que no se puede encontrar en internet ni en ninguna otra parte, sino exclusivamente en el aula de clase.

De igual manera, el adecuado ejercicio del poder docente implica el desarrollo de clases productivas, con temáticas que orientadas desde la planeación, busquen el logro de objetivos o/y metas reales al contexto del estudiante y a las directrices propuestas por el Ministerio de Educación Nacional; una clase implica el desarrollo de competencias entendidas como "la capacidad de utilizar los conocimientos en situaciones distintas de las que se aprendieron. Implica la comprensión del sentido de cada actividad y sus implicaciones éticas, sociales, económicas y políticas" 46.

En la actualidad, el uso de las tecnologías de información y comunicación se convierten en una excelente herramienta educativa, que le permite al docente hacer una clase más atractiva, productiva y didáctica, acorde con los intereses de los estudiantes. La presencia de las tecnologías de información y comunicación en la educación, ha permitido tanto a docentes como estudiantes tener acceso rápido y fácil a la información, la cual al ser utilizada de manera responsable contribuye en el proceso educativo. "Los estudiantes tienen hoy acceso a mucha más información fuera del aula que dentro de ella. Por eso

44. María Espot, eds. La autoridad del profesor, que es la autoridad y como se adquiere (España: Wolters Kluwer, 2006), https://books.google.com.co/books?id=Xm01LOQMu\&pg=PA175\&dq=La+autoridad +del+pr ofesor.+Qu\%C3\%A9+es+la+autoridad+y+c\%C3\%B3mo+se+adquiere+libro\&source=bl\&ots =te4Xp9HfQZ\&sig=7IHoTmU (15 de septiembre de 2014).

45. Entrevista a Docente No. 2, Institución Educativa Simón Bolívar, Samaniego, Nariño, 21 de junio de 2014.

46 República de Colombia, Estándares básicos de competencias en lenguaje, Matemáticas, Ciencias y Ciudadanas (Bogotá: Ministerio de Educación Nacional, 2006), 12. 
más tecnología en la escuela, por sí sola no trae más acceso al conocimiento socialmente significativo y de calidad" 47 .

Así mismo, la presencia de la investigación en el contexto educativo contribuye al desarrollo de clases más significativas; un docente investigador siempre está en la disposición de aprender, de ampliar sus horizontes hacia cosas nuevas y novedosas que quiere compartir con sus estudiantes. La investigación permite que el ejercicio del poder docente cree una interrelación más significativa entre docente y estudiante, pues ella permite la creación de espacios de encuentro por el saber, que sin duda alguna hacen del conocimiento una ciencia transversal, interdisciplinar, eficiente y significativa. "El ejercicio investigativo asumido desde teorías críticas puede dirigir procesos reales de transformación no solo de las prácticas pedagógicas sino de las prácticas sociales inmersas en la Escuela, favoreciendo la democratización del conocimiento y paliando los efectos de la desigualdad social" 48 .

Por lo anterior, se debe tener en cuenta que dentro del ejercicio de la profesión docente uno de los elementos más significativos para lograr el éxito del proceso de enseñanza - aprendizaje, es la preparación de la clase que implica tener una visión global de la temática que se va a explicar, conocer que tanto saben los estudiantes sobre el tema y definir un objetivo a alcanzar, a través de la utilización de diferentes estrategias metodológicas que se apoyan en los medios y métodos más adecuados para alcanzar las metas propuestas.

De esta manera, cada una de las clases desarrolladas por el docente requiere de la más minuciosa preparación, es decir de una planeación, que tenga en cuenta las necesidades de los estudiantes y cumpla con las expectativas de los mismos.

De igual manera, el arte de hablar se constituye en uno de los elementos más utilizados para el ejercicio del poder docente; un discurso objetivo, elocuente y emotivo hace del proceso de enseñanza un proceso más dinamizador. Cuando una clase cae en la monotonía de un discurso retórico que utiliza las mismas palabras, expresiones y tono de voz, se desencadena en el estudiante la falta de motivación, el desinterés a la clase y el bajo rendimiento académico, que lleva a

47. Fernando Savater, Ser maestro hoy: el sentido de educar y el oficio docente (Bogotá: Altablero, 2005), http://www.mineducacion.gov.co/1621/propertyvalues-31232_tablero_pdf.pdf $(20$ de abril de 2014).

48. Marta Osorno. Experiencias docentes, calidad y cambio escolar: investigación e innovación en el aula (Colombia: Grupo Editorial Gaia, 2003), 23. 
que los objetivos de aprendizaje propuestos queden en una meta, que no pasó del papel escrito.

Así mismo, otra de las manifestaciones del ejercicio del poder docente es el manejo de grupo sin el cual al docente se le dificulta la organización de las actividades en aula, el control de los tiempos para la ejecución de tareas u organización de éstas para su correcta realización. Para el docente lograr el manejo de grupo debe alcanzar unos ciertos niveles de reputación, de autoridad, de respeto, es decir, debe proyectar una imagen íntegra que le permita salir airoso de las múltiples y constantes evaluaciones que le hacen los estudiantes: ¿Se expresa correctamente? ¿Viste bien? ¿Sabe o no sabe? ¿Es respetuoso? ¿Trata a todos con igualdad?

En síntesis, para el logro del ejercicio del poder docente con un sentido pedagógico, el docente requiere de ingenio, de dominio de conocimientos, metodologías y técnicas de enseñanza, de paciencia, tolerancia y dominio de las emociones, de tal manera que su actuar en el aula de clase genere un ambiente de convivencia, respeto y disciplina. Características que de igual manera, le permiten al docente desarrollar un proceso evaluativo que más que medir conocimientos permite fortalecerlos y despertar la inquietud por nuevos saberes.

De esta manera, el docente posee un reconocimiento social e institucional que le da una determinada jerarquía, una condición que le permite dentro del proceso evaluativo emitir juicios y valoraciones para formar hábitos, conductas y actitudes en los estudiantes. Así, la evaluación al constituirse en un proceso continuo y permanente, está en constante relación con el ejercicio del poder docente como elemento necesario e inevitable.

\section{Conclusiones}

En la construcción del estado del arte de la investigación sobre "El ejercicio del poder docente en la evaluación de los aprendizajes", se puede concluir que:

La evaluación siempre ha estado en el corazón de los debates educativos, por constituirse en un eje central de la formación de las futuras generaciones y ser un concepto complejo de comprender e importante ampliar, para sacarlo de la reducida semántica de la asignación de una nota o una calificación.

Con respecto al poder, este es una cuestión que persiste en la vida de la sociedad y se ha constituido en un fenómeno que varios autores se han esforzado por comprender, explicar y caracterizar sus implicaciones en la 
vida cotidiana, Weber, Foucault, Bordieu y Arendt, emplean el concepto del poder para comprender la dinámica de las relaciones sociales, explicando que permite la cohesión social, la reproducción de ideologías, simbologías y la base del funcionamiento de los aparatos institucionales, entre otros temas.

En relación, al ámbito educativo el poder no se manifiesta exclusivamente mediante la dominación, sino que existen otras manifestaciones le dan un sentido pedagógico, más humano y productivo al acto de enseñar y al proceso evaluativo. Es en las relaciones entre docente y estudiante, donde subyacen las relaciones de poder, especialmente en el escenario del proceso evaluativo, al ser un proceso continuo y permanente que involucra diferentes aspectos que van desde la preparación de clase, el dominio de conocimientos el manejo de grupo hasta las actitudes y gestos de los docentes.

El ejercicio del poder docente debe ser bien manejado de tal manera que no afecte la autonomía, la autoestima y la identidad de los estudiantes, con la imposición de valores, contenidos y significados que no sean pertinentes. Las acciones y las emociones que se enlazan en el marco del ejercicio del poder, son las que más tiempo permanecen en la memoria de los estudiantes y marcan toda su existencia.

De manera permanente, los docentes ejercen el poder que se deriva de la institución legítima, investida de la función social de enseñar ${ }^{49}$. Por lo tanto, el docente demuestra su autoridad, su capacidad para manejar un grupo, dirigir las conductas, controlar los tiempos, seleccionar contenidos y determinar los instrumentos de evaluación, para a través del ejercicio del poder influir en los procesos de enseñanza- aprendizaje.

49. Bechelloni. "Del análisis de los procesos de reproducción”, en La Reproducción, Bourdieu y Passeron (Barcelona: Fontamara 1996), 288. 


\section{REFERENCIAS BIBLIOGRÁFICAS}

\section{Fuentes primarias}

República de Colombia, Ministerio de Educación Nacional, Decreto 230 de 2002.

República de Colombia, Ministerio de Educación Nacional, Estándares Básicos de Competencias en lenguaje, Matemáticas, Ciencias y Ciudadanas. Bogotá: Ministerio de Educación Nacional, 2006.

República de Colombia, Ministerio de Educación Nacional, Decreto 1290 de 2009.

Arendt, Hanna, eds., Qué es la autoridad parte I (s.c. 2011) http://es.scribd.com/doc/53732515/ArendtHannah-Que-Es-La-Autoridad-Parte-I.

Arendt, Hanna. La condición humana. Barcelona: Paidós Ibérica S. A, 2005.

Arranz, María y Santiuste, Victor, eds. Nuevas perspectivas en el concepto de evaluación. Madrid: Universidad complutense de Madrid (s.f.), http://www.revistaeducacion.mec.es/re350/re350_20.pdf

Bordieu, Pierre y Passeron, Jean Claude. La reproducción: elementos para una teoría de un sistema de enseñanza. México: Fontamara, 1995.

Cajiao Francisco, eds. Plan Decenal en acción: Evaluación del Aprendizaje y Calidad Educativa. Bogotá: Ministerio de Educación Nacional, 2008, http://cms-static.colombiaaprende.edu.co/cache/binaries/articles-158486_archivo2.pdf?binary_rand=4009

Cajiao, Francisco. La formación de maestros y su impacto social. Bogotá: Magisterio, 2004.

Calvache, Edmundo. La evaluación integral en los alumnos de los programas de licenciatura en la Facultad de Ciencias Humanas de la Universidad de Nariño. Tesis especialización en/ Universidad Nariño, 2005.

Carrillo, Liberia; Niño, Libia y Perafán, Andrés, eds., La evaluación: ¿instrumento de poder o acción cultural? (Bogotá: Universidad Pedagógica Nacional, 1996), http://www.pedagogica.edu.co/stogare/ps/articulos/ peda08_05eval.pdf.

Entrevista a Docente No. 01, Institución Educativa Simón Bolívar, Samaniego, Nariño, 21 de junio de 2014

Entrevista a Docente No. 02, Institución Educativa Simón Bolívar, Samaniego, Nariño, 21 de junio de 2014

Entrevista a Docente No. 3, Institución Educativa Simón Bolívar, Samaniego, Nariño, 21 de junio de 2014.

Entrevista a Docente No. 04, Institución Educativa Simón Bolívar, Samaniego, Nariño, 21 de junio de 2014.

Entrevista a Docente No. 5, Institución Educativa Simón Bolívar, Samaniego, Nariño, 21 de junio de 2014.

Entrevista a Docente No. 06, Institución Educativa Simón Bolívar, Samaniego, Nariño, 21 de junio de 2014

Entrevista a Docente No. 8, Institución Educativa Simón Bolívar, Samaniego, Nariño, 21 de junio de 2014.

Espot, María, eds., La autoridad del profesor, que es la autoridad y como se adquiere. España: Wolters Kluwer 2006, https: //books.google.com.co/books?id=Xm01L0QMucMC\&pg=PA175\&lpg=PA175\&dq=La+autorid $\mathrm{ad}+\mathrm{del}+$ profesor. $+\mathrm{Qu} \% \mathrm{C} 3 \% \mathrm{~A} 9+\mathrm{es}+\mathrm{la}+$ autoridad $+\mathrm{y}+\mathrm{c} \% \mathrm{C} 3 \% \mathrm{~B} 3 \mathrm{mo}+\mathrm{se}+$ adquiere+libro\&source=bl\&ots=te 4Xp9HfQZ\&sig=7IHoTmU

Foucault, Michel, eds., "El sujeto y el poder”. Revista Mexicana de Sociología, vol. 50. No. 3, (1988).

Hernández, Carlos, eds., Para ejercer el oficio de maestro. Bogotá: Ministerio de Educación Nacional, 2005, http://www.mineducacion.gov.co/1621/article-87110.html 
Izquierdo Moreno, Ciriaco. El profesor y su mundo. Guías para maestros y profesionales de la educación. México: Editorial Trillas, 2002.

Martínez, O. y Herrera, R. Finalidades y alcances del Decreto 230 del 11 de febrero de 2002. Bogotá: Enlace Editores Ltda., 2002.

Mclaren, Peter. La vida en las escuelas. Madrid, España: Siglo XXI Editores, 1989.

Osorno, Marta. Experiencias docentes, calidad y cambio escolar: investigación e innovación en el aula. Colombia: Grupo Editorial Gaia, 2003.

Ramírez, A. Evaluación de los aprendizajes y desarrollo institucional. Bogotá: ECOE, 2009.

Salazar Cabrales, Omar, eds. "Contexto de la evaluación de los aprendizajes en la educación superior en Colombia: Sugerencias y alternativas para su democratización". Revista Educación y Desarrollo Social. Vol. II - No. 01 (2008).

Sánchez Amaya, Tomas. Aproximación a un estudio genealógico de la evaluación educativa en Colombia, segunda mitad del siglo XX. Tesis Doctoral/ Doctorado en Ciencias Sociales. Niñez y Juventud Universidad de Manizales. 2008.

Sandoval, Carlos. La investigación cualitativa. Bogotá: Arfoeditores, 2002.

Savater, Fernando, eds. Ser maestro hoy: el sentido de educar y el oficio docente. Bogotá: Altablero, 2005, http: //www.mineducacion.gov.co/1621/propertyvalues-31232_tablero_pdf.pdf

Tobón, Sergio. Formación basada en competencias. Bogotá: Ecoe Ediciones Ltda., 2004.

Valletta, María Laura. Diccionario Jurídico. Buenos Aires: Valletta Ediciones, 2006.

Van Dijk, Teun. Ideología una aproximación disciplinaria. Barcelona: Gedisa, 2000.

Vargas, Ana Isabel. "La evaluación educativa: conceptos, periodos y modelos". Actualidades Investigativas en Educación, vol. 4. No 2, (2004).

Weber, Max. Economía y Sociedad. Madrid: Fondo de Cultura Económica, 1964. 